\title{
Current state of active trachoma among elementary school students in the context of ambitious national growth plan: The case of Ethiopia*
}

\author{
Molla Gedefaw ${ }^{1,2 \#}$, Ali Shiferaw ${ }^{1,2}$, Zelalem Alamrew ${ }^{1,2}$, Amsalu Feleke $^{3}$, Tsegaw Fentie ${ }^{3}$, \\ Kiros Atnafu ${ }^{1,2}$ \\ ${ }^{1}$ Joint MPH Program, Bahir Dar University, Bahir Dar, Ethiopia; ${ }^{\#}$ Corresponding Author: mollagedefaw@yahoo.com \\ ${ }^{2}$ GAMBY College of Medical Sciences, Bahir Dar, Ethiopia \\ ${ }^{3}$ University of Gondar, Gondar, Ethiopia
}

Received 25 August 2013; revised 25 September 2013; accepted 17 October 2013

Copyright (C) 2013 Molla Gedefaw et al. This is an open access article distributed under the Creative Commons Attribution License, which permits unrestricted use, distribution, and reproduction in any medium, provided the original work is properly cited.

\section{ABSTRACT}

Trachoma is a classical disease of poverty. It is still the second leading cause of blindness in Ethiopia. Cognizant of the poor living condition of its people, every effort of the current government of Ethiopia is geared towards eradication of extreme poverty. The main aim of this study was to assess the current status and correlates of active trachoma among elementary school students. A cross sectional school-based study was conducted in Dangla town administration in the month of March 2012. Multistage sampling technique was employed. Pre-tested structured questionnaire and eye examination were data collection tools. The data were collected by health professionals especially trained for eye care. The data were analyzed using SPSS version 16 for windows. Possible associations, and statistical significance between, and among variables were measured using odds ratio at $95 \%$ confidence interval. The study indicated that overall prevalence of active trachoma was $12 \%$. The multivariate analysis revealed that those children with unclean face were seven times more likely to develop active trachoma than those with clean face $(A O R=7.07,95 \% \mathrm{Cl}: 3.36-14.96)$. Moreover, children who didn't use soap to wash their face were two times more likely to develop active trachoma than those who claimed to use soap to wash their face (AOR $=2.03,95 \% \mathrm{Cl} 1.72$ - 3.01). In short, the prevalence of trachoma is lower than the figures in previous survey reports

*Conflict of Interest: None of the authors have any conflicts of interest to declare. in the region and at national levels; however, the risk factors remain the same. The struggle to eradicate extreme poverty should continue in a sustainable manner so that diseases of poverty including trachoma could be eliminated altogether.

Keywords: Active Trachoma; School Children; National Plan; Ethiopia

\section{INTRODUCTION}

Trachoma is probably one of the oldest infectious diseases known to mankind. It is caused by Chlamydia trachomatis, and transmitted via contact with eye discharge from the infected person through towels, handkerchiefs, fingers, etc. [1,2].

Globally 1.2 billion people live in trachoma endemic areas [3]. Reports indicate that trachoma is endemic in 55 countries, mainly in Africa and Asia [4]. About 49\% of the global burden of active trachoma is concentrated in five countries: Ethiopia, India, Nigeria, Sudan and Guinea. One of the 2011 reports of WHO [2] indicates that trachoma currently affects about 84 million people worldwide of whom about 8 million are visually impaired.

This disease had been also common in Europe and North America, and virtually disappeared in these continents long before antibiotics were used for its treatment. This phenomenon leads us to believe that the secret behind the disappearance of trachoma in these continents is improvement of living conditions of the people [5]. This in turn supports the widely accepted belief that trachoma is a classical disease of poverty, and hence people living in socioeconomically deprived situations where basic 
needs such as housing, water, and sanitation are failing to carry the major segment of this stubborn disease [1,6].

Ethiopia is one of the least among the low income countries ranking only 157 out of 169 countries in the world [7]. Poverty has undermined its beautiful history, and long standing resistance to colonialism, and its historical heritages. It has been affected by drought and famine repeatedly, and has one of the worst health indicators in the world. Maternal mortality and childhood under nutrition can be mentioned among others [8].

In response to this, and many other multi-dimensional challenges which have been retarding the overall development of the nation, the Government of the Federal Democratic Republic of Ethiopia developed strategies and policies including climate change resilience carbon neutral green economy to challenge these development challenges [9].

Using these modern policies and strategies, the government seems to start its genuine struggle to combat poverty and to develop Ethiopia from its present statusamong the least from low income countries to the status of middle income countries by the year 2025. Following this, one can observe some visible good developmental changes in Ethiopia such as water and sanitation coverage, supply of electricity, primary education enrolment etc. [8].

Trachoma has always been recognized as a serious public health problem in Ethiopia. Several studies were conducted in different parts of the country [10-14]. These pocket studies revealed that the prevalence of active trachoma was among the highest even in the context of Subsahran African Countries.

A national study conducted by Yemane et al. (2007) [15] confirmed the findings of the previous pocket studies. This national study showed that national prevalence of active trachoma (either TF or TI) for children in the age group 1 - 9 years was $40.14 \%$. The study also revealed that there were regional variations: Amhara (62.6\%), Oromia (41.3\%), SNNP (33.2\%), Tigray (26.5\%), Somali $(22.6 \%)$ and Gambella (19.1\%).

In response to this specific public health problem, the Government of the Federal Democratic Republic of Ethiopia signed the Declaration of Support for VISION 2020. It also developed its own 20 years strategic plan to eliminate trachoma. The government acknowledges that eliminating avoidable blindness in Ethiopia by the year 2020 needs great efforts of the government, development partners as well as all affected communities themselves [16].

According to the national strategic plan document, one of the targets of the strategic plan was to reduce active trachoma in the country to less than $10 \%$ at least in $80 \%$ of targeted districts by the year 2010 .

In light of this genuine endeavor of the nation, con- ducting research to update policy makers about current status of trachoma is critical. Hence, this study was conducted in Amhara National Regional State where the highest prevalence of active trachoma was observed in the national survey (2006) [15] to update local decision makers on the current status of active trachoma in school children.

\section{METHODS AND MATERIALS}

A school based cross sectional study was conducted in the month of March 2012. The study was conducted in Dangla town Administration. It is located $485 \mathrm{~km}$ northwest of Addisabeba, the capital of Ethiopia, and $78 \mathrm{~km}$ south of Lake Tana, and Bahir Dar city, the capital of Amhara National Regional State.

The town administration had an estimated population of 34,014. There are 10 governmental elementary schools with a total number of 9101 students. Of these, 4593 were females. One from rural and one from urban schools were randomly selected using simple random sampling technique. The sample size was determined using single population proportion formula $n=Z^{2} p \cdot(1-$ $\mathrm{p}) / \mathrm{w}^{2}$. The proportion of trachoma was determined based on a previous study in the region for Awi Zone [17]. With assumption of level of confidence $95 \%$ at $5 \%$ type I error, the sample size was calculated as follows: $\mathrm{n}=$ minimum sample size, $\mathrm{p}=$ proportion of trachoma in school children, $w=$ maximum error tolerance $(5 \%)$ and $\mathrm{Z}=1.96$.

Then, $\mathrm{n}=(1.96)^{2} \times 0.389 \times(1-0.389) /(0.05)^{2}=$ 365.074. The final calculated sample size with $12 \%$ allowance for non response was 409. This number was then proportionally distributed into two of the selected schools based on the number of students they have. Finally, study participants were selected using computer generated random number.

This study was conducted in school children because active trachoma is very common in small children than any other segment of the population. Children aged 1 - 9 years of age are reservoir of trachoma infection, and active trachoma prevalence studies are recommended to be studied in this age group [18].

Data were collected using structured questionnaire, and eye examination. The questionnaire was adopted from reviewed literature $[15,19]$. It was written in English then translated into Amharic and back to English by language experts.

Eye examination: Each student underwent eye examination by nurses especially trained for eye care. Each eye was examined with the examiner sitting in front of study participants in the day light using a binocular loupe $(\times 2.5)$ and using a hand flash light (torch). Each eye was examined separately. The examiner was cleaning his hands with alcohol between each examination. The di- 
agnosis of trachoma was made based on the WHO five sign grading system [18].

This document briefly and clearly describes the five stages of trachoma.

1) $\mathbf{T F}=$ Trachomatous inflammation-(follicles): five or more follicles, at least $0.5 \mathrm{~mm}$ in size, on the "flat" surface of the upper tarsal conjunctiva.

2) $\mathbf{T I}=$ Trachomatous inflammation-(intense): inflammatory thickening of the upper tarsal conjunctiva with more than half of the normal deep tarsal vessels obscured.

3) TS = Trachomatous scarring: scarring of the tarsal conjunctiva (fibrosis).

4) $\mathbf{T T}=$ Trachomatous trichiasis: at least one eyelash rubbing on the eyeball or evidence of eyelash removal.

5) CO = Corneal Opacity: where at least part of the pupil is blurred or obscured.

Data quality was assured by selecting health workers who were trained and qualified for integrated eye care especially in trachoma diagnosis, grading and treatment including surgery (tarasatomy). Additionally, these data collectors had received several trainings by non-governmental organizations such Carter Center to serve as data collectors for trachoma surveys and hence had been data collectors for several trachoma studies including the national survey in 2005. For this particular study, the selected data collectors also received a one day refresher training inclusive of demonstration of selected pictures of different stages of trachoma with quizzes followed by two days practical exercise at a health center to compare their diagnosis skill using an ophthalmologist as a gold standard. Each data collector then received competency approval letter from the ophthalmologist.

This study obtained Ethical approval from Ethical Review Committee of Bahir Dar University and communicated with regional health bureau before the time of data collection. Letter of permission was obtained from the health bureau, Dangla Woreda health office, and each selected school directors. Treatment was prescribed for those who were diagnosed with active trachoma, and those with trichasis received appointment to come to the nearby health center for further care.

Data were entered into a computer, and analyzed using SPSS version 16 for windows. Backward stepwise regression was fitted to analyze possible association. The strength of association, and statistical significance was measured using odds ratio at $95 \%$ confidence intervals.

\section{RESULTS}

A total of 409 elementary school children participated in the study. Of them, 215 (52.6\%) were males. Two hundred twenty five $(55 \%)$ were within the age group between $7-10$ years and $184(45 \%)$ were $11-15$ years of age (Table 1).

As shown in Table 2, overall prevalence of active trachoma among primary school children in Dangla administrative town was $12 \%$.

Of the total 225 children of aged between 7 - 10 years, $18(8 \%)$ had active trachoma while the figure was 31 (16.8\%) among children aged between $11-15$ years. As shown in Table 3, the study also indicated that the distribution of active trachoma by sex was $23(10.7 \%)$ in males and $26(13.4 \%)$ in females.

As shown in Table 4, prevalence of active trachoma

Table 1. Socio-demographic characteristics of elementary school children in Dangla town administration, March 2012.

\begin{tabular}{ccc}
\hline Variable & $\begin{array}{c}\text { Number of ex } \\
\text { Examined children }\end{array}$ & Percent \\
\hline Age & & \\
$7-10$ years & 255 & $55 \%$ \\
$11-15$ years & 184 & $45 \%$ \\
Sex & & \\
Male & 215 & $52.6 \%$ \\
Female & 194 & $47.4 \%$ \\
Students residence & & \\
Urban & 234 & $57.2 \%$ \\
Rural & 175 & $42.8 \%$ \\
Students grade & & \\
1 - 4 & 273 & $33.3 \%$ \\
5 - 6 & 136 & \\
Educational status & & \\
Not able to read and write & 92 & $63.6 \%$ \\
Read and write & 57 & \\
Primary and above & 60 & \\
\hline
\end{tabular}

Table 2. Prevalence of active trachoma among primary school children in Dangla town administration by WHO trachoma grading system, March 2012.

\begin{tabular}{ccc}
\hline Active trachoma & Frequency & Percent \\
\hline TF & 22 & $5.4 \%$ \\
TI & 24 & $5.9 \%$ \\
TF and TI & 03 & $0.7 \%$ \\
Total & 49 & $12 \%$ \\
\hline
\end{tabular}

TF: trachomatous inflammation (follicles); TI: trachomatous inflammation (intense) [18].

Table 3. Distribution of active trachoma by age and sex among primary school children in Dangla town administration, March 2012.

\begin{tabular}{cccc}
\hline \multirow{2}{*}{ Sex } & \multirow{2}{*}{ Age } & \multicolumn{2}{c}{ Trachoma } \\
\cline { 3 - 4 } & & Yes & No \\
\hline \multirow{2}{*}{ Male } & $7-10$ & $9(8.1 \%)$ & $102(91.9 \%)$ \\
& $11-15$ & $14(13.5 \%)$ & $90(86.5 \%)$ \\
Female & $7-10$ & $9(7.9 \%)$ & $105(92.1 \%)$ \\
& $11-15$ & $17(21.2 \%)$ & $63(78.8 \%)$ \\
\hline
\end{tabular}


Table 4. Prevalence of active trachoma by selected variables among primary school children in Dangla administrative town, March, 2012.

\begin{tabular}{ccc}
\hline \multirow{2}{*}{ Variables } & \multicolumn{2}{c}{ Trachoma } \\
\cline { 2 - 3 } & Yes & No \\
\hline Grades & $23(8.4 \%)$ & $250(91.6 \%)$ \\
$1-4$ & $26(19.1 \%)$ & $110(80.9 \%)$ \\
$5-6$ & & \\
Face cleanliness & $18(8.5 \%)$ & $332(91.5 \%)$ \\
Clean & $31(39.1 \%)$ & $28(60.9 \%)$ \\
unclean & & \\
Soap use & $10(5.1)$ & $186(94.9)$ \\
Yes & $39(18.3)$ & $174(81.7)$ \\
no & & \\
Residences & $17(7.3 \%)$ & $217(92.7 \%)$ \\
Urban & $32(18.3 \%)$ & $143(81.7 \%)$ \\
Rural & &
\end{tabular}

among children who had clean face was $8.5 \%$ while this figure was $39.1 \%$ among children with unclean face. Those Children claimed to use soap for face washing were at lower risk of developing active trachoma than those who did not use (5.1\% vs. $18.3 \%$ ).

The multivariate analysis result indicated that children aged between $11-15$ years were 2.77 times more likely to develop active trachoma than those whose age lies between 7 - 10 years (AOR $=2.77,95 \%$ CI: $1.42-5.43$ ). As shown in Table 5, children with unclean face were seven times more likely to develop active trachoma than those children with clean face (AOR $=7.07,95 \%$ CI: 3.36 - 14.96). Besides, children who didn't use soap to wash their face were more than two times more likely to develop active trachoma than those who claimed to use soap to wash their face (AOR $=2.03,95 \%$ CI 1.718 3.009).

\section{DISCUSSION}

The major objective of this study was to update the prevalence of active trachoma, and to identify associated factors among school children. In this study, the overall prevalence of active trachoma was lower than the findings from other studies in the region, and other regions of Ethiopia $[15,17,20]$. The latest of the prevalence study reports in the region revealed that prevalence of active trachoma be about $24 \%$ [21].

According to the present study, the prevalence of active trachoma among children aged between 7 - 10 years was $8 \%$. This figure is probably the smallest figure ever recorded in the studies conducted in the region. This might herald reduction of transmission of trachoma in the community.

This finding is further consolidated by the fact that the multivariate logistic regression analysis revealed that
Table 5. Factors associated with the prevalence of active trachoma among elementary school children in Dangla town administration, March 2012.

\begin{tabular}{|c|c|c|c|c|}
\hline \multirow{2}{*}{ Variables } & \multicolumn{2}{|c|}{ Trachoma } & \multirow{2}{*}{ COR } & \multirow{2}{*}{ AOR (95\% CI) } \\
\hline & Yes & No & & \\
\hline \multicolumn{5}{|l|}{ Age } \\
\hline $7-10$ years & 18 & 207 & 1.00 & 1.00 \\
\hline $11-15$ years & 31 & 153 & $2.33(1.25,4.31)$ & $2.777(1.42,5.43)$ \\
\hline \multicolumn{5}{|l|}{ Sex } \\
\hline Male & 23 & 192 & 1.00 & 1.00 \\
\hline Female & 26 & 168 & $1.29(0.710,2.35)$ & $1.46(0.76,2.84)$ \\
\hline \multicolumn{5}{|l|}{ Residence } \\
\hline Urban & 17 & 217 & 1.00 & 1.00 \\
\hline Rural & 32 & 143 & $2.86(1.529,5.34)$ & $1.568(0.745,3.301)$ \\
\hline \multicolumn{5}{|l|}{ Waste disposal } \\
\hline Burning/burying & 41 & 310 & 1.00 & 1.00 \\
\hline Open field & 8 & 50 & $1.21(0.54,2.73)$ & $1.83(0.72,4.65)$ \\
\hline \multicolumn{5}{|l|}{ Household } \\
\hline With latrine & 45 & 339 & 1.00 & 1.00 \\
\hline Without latrine & 4 & 21 & $1.44(0.471,4.37)$ & $1.21(0.292,5.04)$ \\
\hline \multicolumn{5}{|l|}{ Cattle owner } \\
\hline Yes & 32 & 201 & $1.49(0.798,2.79)$ & $1.55(0.78,3.09)$ \\
\hline No & 17 & 159 & 1.00 & 1.00 \\
\hline \multicolumn{5}{|l|}{ Facial cleanliness } \\
\hline Clean face & 31 & 332 & 1.00 & 1.00 \\
\hline Un clean face & 18 & 28 & $6.89(3.43,13.83)$ & $7.09(3.36,14.96)$ \\
\hline \multicolumn{5}{|l|}{ Soap use } \\
\hline Yes & 10 & 186 & 1.00 & 1.00 \\
\hline No & 39 & 174 & $4.17(1.01,5.44)$ & $2.03(1.72-3.01)$ \\
\hline
\end{tabular}

children aged between 11 and 15 years were nearly three times more likely to have active trachoma than those children aged between 7 - 10 years. Normally, active trachoma is very common in young children aged between 1 - 9 years [18]. Therefore, this finding cannot be an artifact. It most probably indicates that elder children (11 - 15) had been repeatedly infected while they were young than those children aged $7-10$ years because transmission has been reduced following several interventions which are going on in the study area.

The prevalence of trachoma in the present study is also lower than the WHO threshold level used to determine trachoma as a severe public health problem. According to WHO, the threshold prevalence for TF/TI among children age $1-10$ years old is $20 \%$ [22].

Several factors might have contributed to the observed reduction of the prevalence of active trachoma in the present study. Three of them might be worth mentioning here. They are following:

1) Implementation of Health Extension Packages with 16 components, a special program integrated to the primary health care system of Ethiopia designed to increase health care coverage and to empower communities to prevent classical poverty related diseases including trachoma. 
2) Trachoma specific interventions such as single annual dose Azithromax distribution.

3) Overall improvement of water and sanitation coverage in the region.

This study also showed that unclean face was significantly associated with the prevalence of active trachoma $(\mathrm{AOR}=7.09,95 \% \mathrm{CI}: 3.35-14.96)$. This is in accordance with previous researches in the country $[15,19$, 21].

Furthermore, this study revealed that washing face with soap was one of the significant predictors of active trachoma among children. Children who claimed to use soap during face wash were more than two times more likely to develop trachoma than children never used soap to wash face. This is in accordance with other evidences where ensuring facial and hand cleanliness using water and soap to keep children's face clean throughout the day are important component of trachoma prevention [23]. Unclean face attracts flies, and flies are important mechanical vectors for the transmission of trachoma [24].

There are other factors which are found to have statistically significant association with presence of active trachoma in the present study. These include rural residence, open field waste disposal, and absence of latrine. These factors are directly and indirectly mentioned as risk factors in several documents. For instance, Center for International Health (2007) [24] identifies six Ds to make memorization of risk factors of trachoma easier. Dry, Dusty, Dirty, Dung, Discharge, Density, (overcrowding in the home).

The odd of having active trachoma in females was higher than in males. Logically, this can be partly explained by the roles of female children in the household-taking care of small children, cleaning the house, cooking etc. which might increase risk of acquiring trachoma infection.

All these indicate that trachoma is disease of the poor that could be eliminated by simple hygienic measures. Trachoma was once a public health problem in the western world-high income countries. Evidences indicate that these countries eliminate trachoma and other classical poverty diseases by improving the living condition of the citizens-housing, basic sanitation, and nutrition $[1,5,6]$.

Unfortunately, however, trachoma still imposes serious health, social, and economic burden in Ethiopia. Ethiopia is one of the most climate change vulnerable countries in Africa and the world at large while it is geographically located at the centre of the Nile River which supports the livelihoods of 160 million people in the Eastern and Horn of Africa. The country has been affected by drought and famine several times [7].

Cognizant of this challenge, the current government of the Federal Democratic Republic of Ethiopia is involved in mammoth undertakings to fighting poverty-old age enemy of the country. The major development agenda of the nation is poverty eradication to make the prevailing extreme poverty history [25]. The great blue Nile Dam is one of the examples of the mammoth undertakings for Ethiopia. Double digit economic growth has been documented in Ethiopia since some years amidst of world Economic crises [26].

In fact, the choice of Ethiopia is not merely economic growth, and eradication of poverty. Its choice of development path is one of the most modern, and attractive to its people and the world community at large. Its choice is to become one of the middle income countries in the world by the year 2025 through climate change resilience carbon neutral green economy strategy [9].

The government is striving to make basic health care universally accessible to all citizens. The struggle of the nation to make water and basic sanitation accessible to all citizens is also appreciable [8]. Moreover, the government of Ethiopia works very closely with development partners such as Carter Center, World Vision, and Lions club to eliminate trachoma [16]. These concerted efforts might have contributed for the possible reduction of active trachoma in the study area.

This school based cross sectional study suffers from all the limitations of institution based studies. Firstly, it is not a community based study so its external validity could be narrow. However, Ethiopia has made tremendous improvement in primary education enrolment most children in the region visit school currently so that the finding can be generalized to similar setups in the region. Secondly, it is a snap short survey study, hence, logically it can tell nothing about what was the situation some time ago or what will it be some time after the data collection. However, trachoma, unlike acute childhood diarrhea, is a chronic disease so that one can safely guess based on the findings of this study about the situations before and after the survey for a reasonable period of time, we believe.

\section{CONCLUSION}

The prevalence of active trachoma in the present study is the smallest ever recorded in the region. This might herald that trachoma transmission is decreasing in the community. However, Ethiopian children still suffer from trachoma of high magnitude like their parents as if it was a genetic disease just because water and soap are not available to them. The struggle to eradicate extreme poverty should continue in a sustainable manner so that poverty related diseases such as trachoma could be eliminated altogether. This time, Ethiopia can do that providing that peace in the horn of Africa is maintained and the world community welcomes its modern development policies. 


\section{ACKNOWLEDGEMENTS}

We are very grateful to Bahir Dar University and GAMBY College of Medical Sciences for opening the joint MPH program. Our heartfelt appreciation goes also to study participants and the school management.

\section{REFERENCES}

[1] A Safe Solution (2011) Sight savers' fast track initiative to eliminate blinding trachoma.

[2] WHO (2011) Prevention of blindness and visual impairment: Priority eye diseases.

[3] Mariotti, S.P., Pascolini, D. and Rose-Nussbaumer, J. (2008) Trachoma: Global magnitude of a preventable cause of blindness. British Journal of Ophthalmology, 93, 563568.

[4] Polack, S., Brooker, S., Kuper, H., Mariotti, S., Mabey, D., and Foster, A. (2005) Mapping the global distribution of trachoma. Bulletin of the World Health Organization, 83, 913-919.

[5] Mariotti, S.P., Pararajasegaram, R. and Resnikoff, S. (2003) Trachoma: Looking forward to global elimination of trachoma by 2020 (GET 2020). The American Journal of Tropical Medicine and Hygiene, 69, 33-35.

[6] Roodhoff, J.M.J. (2002) Leading causes of blindness worldwide. Bulletin de la Societe Belge d'Ophtalmologie, 283, 19-25.

[7] UNFCCC (2009) Climate change impacts, vulnerabilities and adaptation in developing countries. Regional impacts and vulnerabilities to climate change in Africa.

[8] Ethiopian Central Statistical Agency and ICF International (2012) 2011 Ethiopia demographic health survey: Key findings. CSA \& ICF International, Calverton.

[9] Ethiopia's Climate-Resilient Green Economy (2010) Green economy strategy. Federal Democratic Republic of Ethiopia I, Addis Ababa.

[10] Zerihun, N. (1997) Trachoma in Jimma zone, south western Ethiopia. Tropical Medicine \& International Health, 2, 1115-1121. http://dx.doi.org/10.1046/j.1365-3156.1997.d01-211.x

[11] Alene, G.D. and Abebe, S. (2000) Prevalence of risk factors for trachoma in a rural locality of north-western Ethiopia. East African Medical Journal, 77, 308-312.

[12] Assefa, T., Argaw, D., Foster, A. and Schwartz, E. (2001) Results of trachoma rapid assessment in 11 village of South Gonder Zone, Ethiopia. Tropical Doctor, 31, 202204.

[13] Alemayehu, W., Malese, M., Fredlander, E., Worku, A. and Courtright, P. (2005) Active trachoma in children in central Ethiopia: Association with altitude. Transactions of the Royal Society of Tropical Medicine \& Hygiene, 99, 840-843. http://dx.doi.org/10.1016/j.trstmh.2005.06.013

[14] Cumberland, P., Hailu, G. and Todd, J. (2005) Active trachoma in children aged three to nine years in rural communities in Ethiopia: Prevalence, indicators and risk factors. Transactions of the Royal Society of Tropical Medicine \& Hygiene, 99, 120-127. http://dx.doi.org/10.1016/j.trstmh.2004.03.011

[15] Berhane, Y., Worku, A., Bejiga, L., Adamu, W., Alemayehu, A., et al. (2007) Prevalence of trachoma in Ethiopia. The Ethiopian Journal of Health Development, 21, 211215.

[16] National Five-Year Strategic Plan for Eye Care in Ethiopia (2006-2010) (2006) Diseases Prevention and Control Department Federal Ministry of Health, Addis Ababa.

[17] The Carter Center (2007) Prevalence of malaria and trachoma in Ethiopia. Household Cluster Survey.

[18] WHO (2006) Trachoma control. A guide for program managers.

[19] Ngondi, J., Gebre, T., Shargie, E.B., Gravesa, P.M, Ejigsemahu, Y., Teferi, T., et al. (2008) Risk factors for active trachoma in children and trichiasis in adults: A household survey in Amhara National Regional State, Ethiopia. Transactions of the Royal Society of Tropical Medicine and Hygiene, 102, 432-438. http://dx.doi.org/10.1016/j.trstmh.2008.02.014

[20] Berta, E. (2004) Prevalence and risk factors of active trachoma among children of rural south Gondar, Ethiopia. MPH Thesis, Addis Ababa University, Ethiopia.

[21] Ketema, K., Tiruneh, M., Woldeyohannes, D. and Muluye, D. (2012) Active trachoma and associated risk factors among children in Baso Liben District of East Gojjam, Ethiopia. BMC Public Health, 12, 1105. http://dx.doi.org/10.1186/1471-2458-12-1105

[22] Bailey, R. and Lietman, T. (2001) The SAFE strategy for the elimination of trachoma by 2020: Will it work? Bulletin of the World Health Organization, 79, 233-236.

[23] Stephanie, O. and Paul, E. (2012) How communities can control trachoma without a big budget. Journal of Community Eye Health, 25, 80-81.

[24] International Center for Eye Health (2007) London School of Hygiene \& Tropical Medicine, UK.

[25] Growth and Transformation Plan (2010) Federal democratic republic of Ethiopia. Volume 1, Ministry of Finance and Economic Development, Addis Abeba.

[26] Ethiopia United Nations Development Assistance Framework 2012 to 2015 (2011) United Nations Country Team. 